\title{
A Nail-size Piezoelectric Energy Harvesting System Integrating a MEMS Transducer and a CMOS Interface Circuit
}

\author{
Sijun Du, Member, IEEE, Yu Jia, Member, IEEE, Chun Zhao, Member, IEEE, Gehan A. J. Amaratunga \\ and Ashwin A. Seshia, Senior Member, IEEE
}

\begin{abstract}
Piezoelectric vibration energy harvesting has drawn much interest to power distributed wireless sensor nodes for Internet of Things (IoT) applications where ambient kinetic energy is available. For certain applications, the harvesting system should be small and able to generate sufficient output power. Standard rectification topologies such as the full-bridge rectifier are typically inefficient when adapted to power conditioning from miniaturized harvesters. Therefore, active rectification circuits have been researched to improve overall power conversion efficiency, and meet both the output power and miniaturization requirements while employing a MEMS harvester. In this paper, a MEMS piezoelectric energy harvester is designed and cointegrated with an active rectification circuit designed in a CMOS process to achieve high output power for system miniaturization. A MEMS energy harvester of $0.005 \mathrm{~cm}^{3}$ size, co-integrated with the CMOS conditioning circuit, outputs a peak rectified DC power of $40.6 \mu \mathrm{W}$ and achieves a record DC power density of $8.12 \mathrm{~mW} / \mathrm{cm}^{3}$ when compared to state-of-the-art harvesters.
\end{abstract}

Index Terms-Keywords: Energy harvesting, MEMS, energy conversion, piezoelectric transducer, rectification, power conditioning.

\section{INTRODUCTION}

The Internet of Things (IoT) continues to expand in both number and variety and the deployment of wireless sensor networks (WSN) has been developed to interconnect the physical and cyber worlds. Harvesting ambient vibration energy for self-powered wireless sensors has received much research interest in recent years [1]-[11]. Among the various vibration energy harvesters, piezoelectricity has been widely used due to its high power density and integration with conventional CMOS circuits [12]-[14]. During real-world implementations, miniaturization of the entire integrated energy harvesting system has been one of the key challenges. In addition, the output power from miniaturized harvesters also need to meet requirements to continuously power the sensors and periodically

$\mathrm{S}$. Du is with the Department of Engineering, University of Cambridge, Cambridge, CB2 1PZ, UK, and also with the Department of Electrical Engineering and Computer Sciences, University of California at Berkeley, Berkeley, CA, 94720, USA. (e-mail: sijundu@hotmail.com; sijun@eecs.berkeley.edu).

Y. Jia is with the Department of Mechanical Engineering, University of Chester, Chester, $\mathrm{CH} 2$ 4NU, UK, and also with the Department of Engineering, University of Cambridge, Cambridge, CB2 1PZ, UK.

G. A. J. Amaratunga and A. A. Seshia are with the Department of Engineering, University of Cambridge, Cambridge, CB2 1PZ, UK.

C. Zhao is with the Department of Engineering, University of Cambridge, Cambridge, CB2 1PZ, UK, and also with the Center for Gravitational Experiments, Huazhong University of Science and Technology, Wuhan, 430074, China. send wireless data [15]-[17]. However, output power and the generated voltage from a miniaturized piezoelectric transducer (PT) are usually much lower than macroscopic PTs. Hence an efficient interface circuit is needed to increase the power extraction efficiency while ensuring that miniaturization benefits are not compromised [18], [19]. A complete vibration energy harvesting system consists of a PT and an interface circuit, which rectifies, manages and regulates the harvested energy to provide a stable DC power supply for load electronics [20]. An system block diagram is shown in Fig. 1a and a miniaturized energy harvesting prototype is fabricated and shown in Fig. 1b. The size of the prototype is $1 \mathrm{~cm} \times 1 \mathrm{~cm} \times 0.4 \mathrm{~cm}$ and it consists of a cantilevered MEMS (Microelectromechanical Systems) PT, a CMOS interface circuit, an inductor and several capacitors.

In order to rectify the raw power generated from a PT, a fullbridge rectifier (FBR) is widely employed due to its simplicity and stability [20], [21]. Fig. 2a shows the circuit diagram of a full-bridge rectifier and the associated waveforms. The PT while vibrating is modeled as a current source $I_{P}$ in parallel with a capacitor $C_{P}$. Power rectification is typically achieved by a FBR, which consists of four diodes, into a storage capacitor $C_{S}$. The signal $V_{P T}$ in the waveform is the voltage across the PT. From the waveform, it can be seen that $V_{P T}$ needs to attain either $V_{S}+2 V_{D}$ or $-\left(V_{S}+2 V_{D}\right)$ in order to transfer energy into $C_{S}$, where $V_{D}$ is the forward voltage drop of the diodes. Hence, $V_{P T}$ needs to be flipped between these two voltage levels for each half period and the energy used to flip $V_{P T}$ is wasted, which is illustrated as fully shaded areas in the $I_{P}$ graph. Assuming the open-circuit voltage amplitude generated from the PT is $V_{O C}$, the condition for a FBR to start operating is:

$$
V_{O C}>V_{S}+2 V_{D}
$$

If equation (1) is not satisfied, all generated energy is wasted (internally dissipated in the harvester front-end). Even if it is marginally satisfied, most of energy is wasted and the power efficiency is extremely low in this case. A FBR may have acceptable power efficiency while employing a macroscopic PT, which can generate relatively high open-circuit voltage. However, MEMS (microelectromechanical system) energy harvesters have been widely employed for miniaturization in the past decade [22]-[25]. if a MEMS PT is employed, the generated voltage can be much lower. For example, assuming 


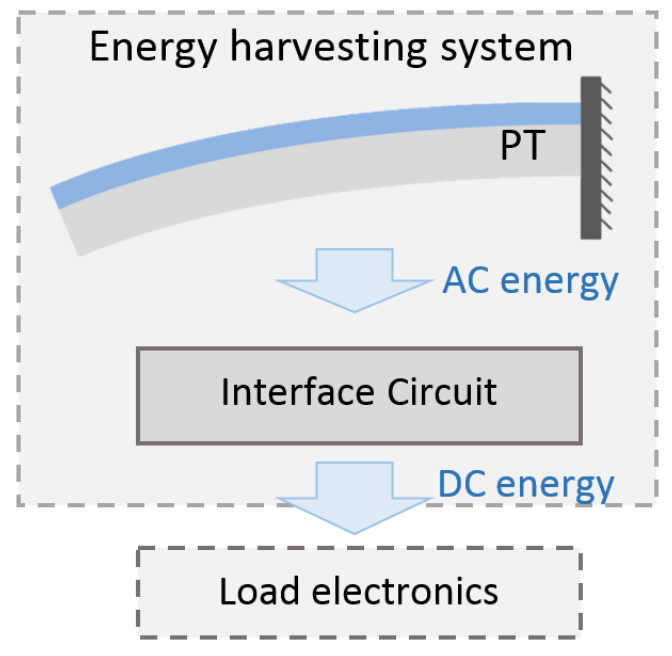

(a)

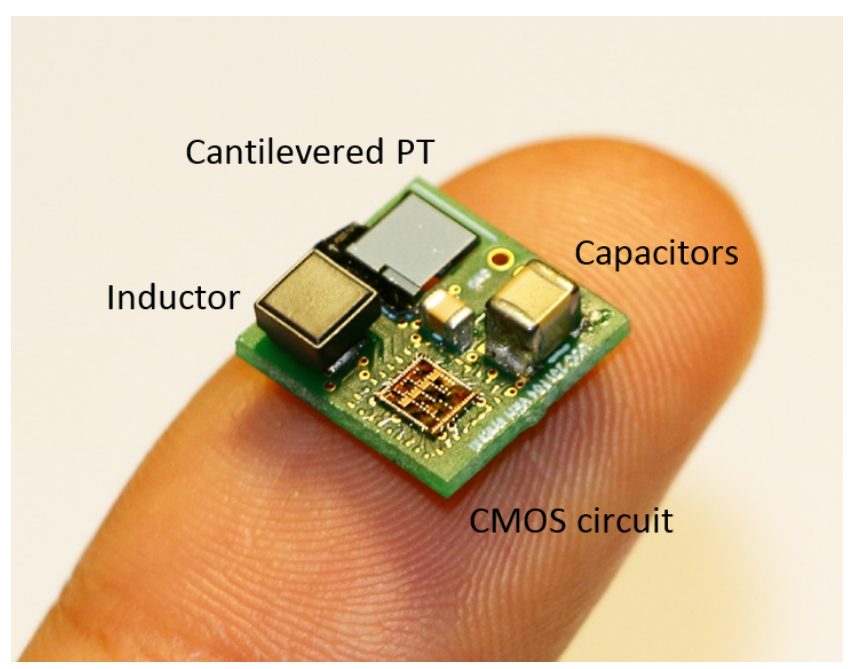

(b)

Fig. 1: System block diagram and a fabricated prototype of a complete vibration energy harvesting system (a) system block diagram (b) prototype of size $1 \mathrm{~cm} \times 1 \mathrm{~cm} \times 0.4 \mathrm{~cm}$.

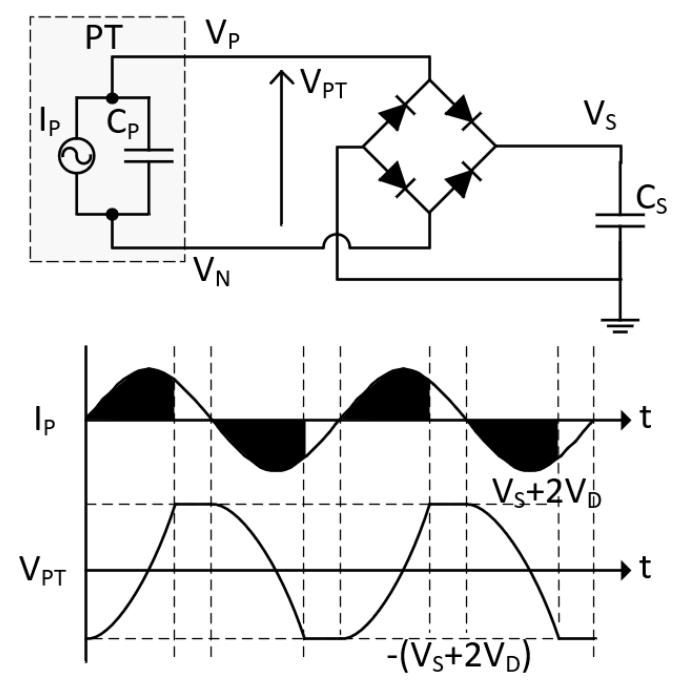

(a)

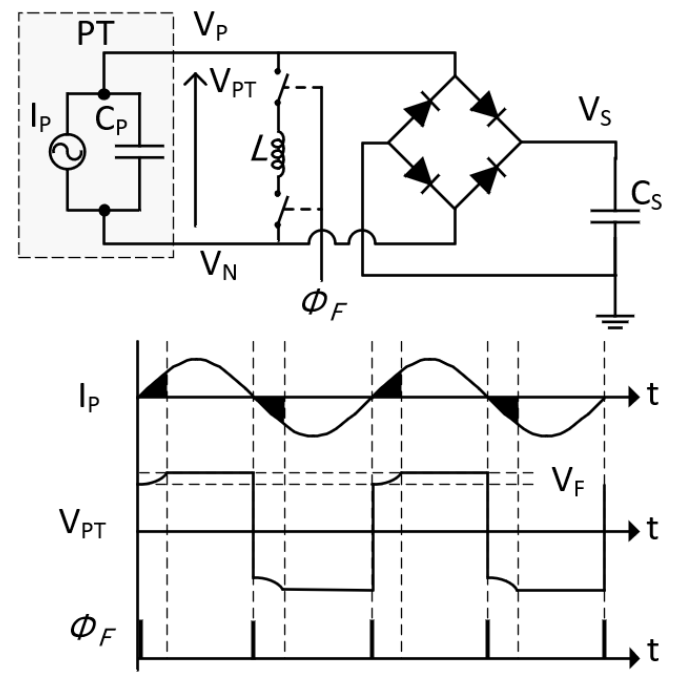

(b)

Fig. 2: (a) Full-bridge rectifier and associated waveforms. (b) SSHI circuit and associated waveforms.

$V_{S}=3 \mathrm{~V}$ and $V_{D}=0.3 \mathrm{~V}, V_{O C}$ needs to attain $3.6 \mathrm{~V}$ (or $7.2 \mathrm{~V}$ peak-to-peak) to be able to turn on the diodes, which can be difficult to achieve for a MEMS PT at low vibration levels. Therefore, in order to increase the extracted power, many active interface circuits have been proposed in recent years [26]-[36]. Most of these circuits employ inductors and synchronous switches to perform nonlinear energy extraction, such as synchronized switch harvesting on inductor (SSHI), synchronous electric charge extraction (SECE), etc [37]-[45].

In this paper, a MEMS PT is fabricated and integrated with a SSHI interface circuit implemented in a $0.35 \mu \mathrm{m}$ CMOS process. The system shows a significant performance improvement while using the SSHI circuit on the MEMS harvester compared with using a FBR rectifier and it provides useful rectified DC power and system integration in a compact volume $\left(0.4 \mathrm{~cm}^{3}\right)$.

\section{OUTPUT POWER MODELING}

\section{A. Full-bridge rectifier}

This section models the full-bridge rectifier (FBR) and analyzes the the DC power transferred into the capacitor $C_{S}$. The circuit diagram of a FBR is shown in Fig. 2a. While the PT is vibrating, the current source can be expressed as $I_{P}=I_{0} \sin (\omega t)$, where $\omega$ is the excitation frequency. Hence, the charge generated in a half period is:

$$
Q_{\text {total }}=\int_{0}^{\frac{T}{2}} I_{0} \sin \omega t \mathrm{~d} t=\frac{2 I_{0}}{\omega}
$$

If the PT is in an open circuit, the open-circuit zero-peak amplitude can be calculated as:

$$
V_{O C}=\frac{1}{2} \frac{Q_{t o t a l}}{C_{P}}=\frac{I_{0}}{\omega C_{P}}
$$


As a certain amount of charge is wasted to flip $V_{P T}$ between $V_{S}+2 V_{D}$ and $-\left(V_{S}+2 V_{D}\right)$, the remaining charge transferred into $C_{S}$ can be expressed as:

$$
Q_{F B R}=Q_{t o t a l}-2\left(V_{S}+2 V_{D}\right) C_{P}
$$

Hence, the energy flowing into $C_{S}$ is:

$$
E_{F B R}=V_{S} Q_{F B R}=2 C_{P} V_{S}\left(V_{O C}-V_{S}-2 V_{D}\right)
$$

Therefore, the extracted power in this half period is:

$$
P_{F B R}=\frac{E_{F B R}}{T / 2}=4 C_{P} V_{S} f_{P}\left(V_{O C}-V_{S}-2 V_{D}\right)
$$

It can be found that the maximum power point (MPP) of $P_{F B R}$ is obtained when $V_{S}=\frac{V_{O C}}{2}-V_{D}$. The peak power transferred to $C_{S}$ is:

$$
P_{F B R(\max )}=4 C_{P} f_{P}\left(\frac{V_{O C}}{2}-V_{D}\right)^{2}
$$

Assuming the forward voltage drop of the diodes, $V_{D}$, is ignorable, the peak power can be rewritten as:

$$
P_{F B R(\max )}=C_{P} f_{P} V_{O C}^{2}
$$

\section{B. SSHI circuit}

In order to minimize the wasted charge due to flipping the voltage $V_{P T}$ across the PT, the synchronized switch harvesting on inductor (SSHI) interface circuit has been proposed and implemented to increase the power extraction efficiency [44]. The circuit diagram and the associated waveforms are shown in Fig. 2b. In the SSHI rectifier, an inductor is employed to form an RLC oscillation loop to flip $V_{P T}$. The pulse signal $\phi_{F}$ is synchronously generated and its pulsewidth is adjusted to be a half-pseudo period of the RLC system. During the pulse $\phi_{F}, V_{P T}$ is flipped with a loss $V_{F}$, which can be expressed as:

$$
V_{F}=\left(V_{S}+2 V_{D}\right)\left(1-e^{\left.-\frac{\pi}{\sqrt{\frac{4 L}{R^{2} C_{P}}-1}}\right)}=\left(V_{S}+2 V_{D}\right) \eta_{F}\right.
$$

where the threshold $V_{F}$ is illustrated in Fig. 2b, which represents the voltage loss after one flip. $R$ is the total resistance in the RLC loop, which consists of the DC resistance of the inductor, the ON resistance of switches and other parasitic resistance in wires and contacts. $\eta_{F}$ is the voltage loss ratio between 0 and 1 and it is expressed as $\eta_{F}=1-e^{-\frac{\pi}{\sqrt{\frac{4 L}{R^{2} C}-1}}}$. As $V_{F}$ is the voltage loss after one flip in a half period, the remaining charge flowing into $C_{S}$ is:

$$
Q_{S(S S H I)}=Q_{t o t a l}-C_{P} V_{F}=C_{P}\left(2 V_{O C}-V_{F}\right)
$$

Hence, the extracted power is:

$$
\begin{aligned}
P_{S S H I} & =2 f_{P} C_{P} V_{S}\left(2 V_{O C}-V_{F}\right) \\
& =2 f_{P} C_{P} V_{S}\left(2 V_{O C}-\left(V_{S}+2 V_{D}\right) \eta_{F}\right)
\end{aligned}
$$

The power attains its MPP when $V_{S}=\frac{V_{O C}}{\eta_{F}}-V_{D}$ and the maximum power can be calculated as:

$$
P_{S S H I(\max )}=2 C_{P} f_{P} \eta_{F}\left(\frac{V_{O C}}{\eta_{F}}-V_{D}\right)^{2}=\frac{2}{\eta_{F}} C_{P} f_{P} V_{O C}^{2}
$$

where $V_{D}$ is ignored. Comparing the MPP values in equations (8) and (12), the performance improvement of using a SSHI circuit compared to a FBR can be expressed as:

$$
\frac{P_{S S H I}}{P_{F B R}}=\frac{2}{\eta_{F}}
$$

It can be found that the performance improvement does not depend on excitation level $\left(V_{O C}\right)$, but $\eta_{F}$. Hence, we need to increase $L$ or decrease $C_{P}$ or $R$ to obtain a higher performance from a SSHI circuit. The inductance $L$ can be increased in a wide range of inductor selections; however, the DC resistance on an inductor should always be considered since larger $L$ in a given volume usually introduces higher DC resistance. The resistance $R$ can be decreased from the inductor selection and the design of the circuits, especially the analogue switches controlling the inductor shown in Fig. 2b. The $C_{P}$ is the inherent capacitance of the PT; hence, it can only be deceased during MEMS design.

\section{SSHI CIRCUIT IMPLEMENTATIONS}

This section presents the circuit implementation of a SSHI rectifier and the system architecture is shown in Fig. 3, which consists of a zero-crossing detection block, a pulse generation block and two analog switches. The full bridge rectifier is formed by four off-chip Schottky diodes and the forward voltage drop is around $V_{D} \approx 0.3 \mathrm{~V}$. The zero-crossing block aims to find the zero-current moment of $I_{P}$, which is the right moment to flip $V_{P T}$. In order to find this moment, two continuous-time comparators are employed to compare the both electrodes of the PT, $V_{P}$ and $V_{N}$, with a reference voltage $V_{\text {ref }}$. While $I_{P}$ is close to zero, the diodes of the FBR are just about to turn OFF. At this moment, one of $V_{P}$ and $V_{N}$ is close to $-V_{D}$ and the other one is close to $V_{S}+V_{D}$. The reference voltage $V_{\text {ref }}$ is set slightly higher than $-V_{D}$ to detect the moment while one of $V_{P}$ and $V_{N}$ leaves $-V_{D}$ and this is the moment while $I_{P}$ is close to zero. The outputs of the two comparators are ANDed and the signal $S Y N$ is a synchronous signal which consists of a rising edge at each zero $I_{P}$ moment. A pulse signal $\phi_{F}$ is then generated according to each rising edge of $S Y N$ to control the analog switches to flip $V_{P T}$. The pulse of $\phi_{F}$ should be adjusted to be a half pseudo-period of the RLC oscillation system to achieve the maximum voltage flipping efficiency.

The pulse generation block aims to generate the fixed-width pulse signal, $\phi_{F}$ from $S Y N$ and the circuit diagram is shown in Fig. 3.This pulse generator is a AND gate where the signal $S Y N$ is ANDed with the delayed and inverted version of itself. The delay is achieved using two weak inverters charging up capacitors. The total capacitance formed by 6 on-chip capacitors can be adjusted by a 6-bit signal controlling the six switches $C_{5}$ to $C_{0}$. This 6-bit signal can be set externally 


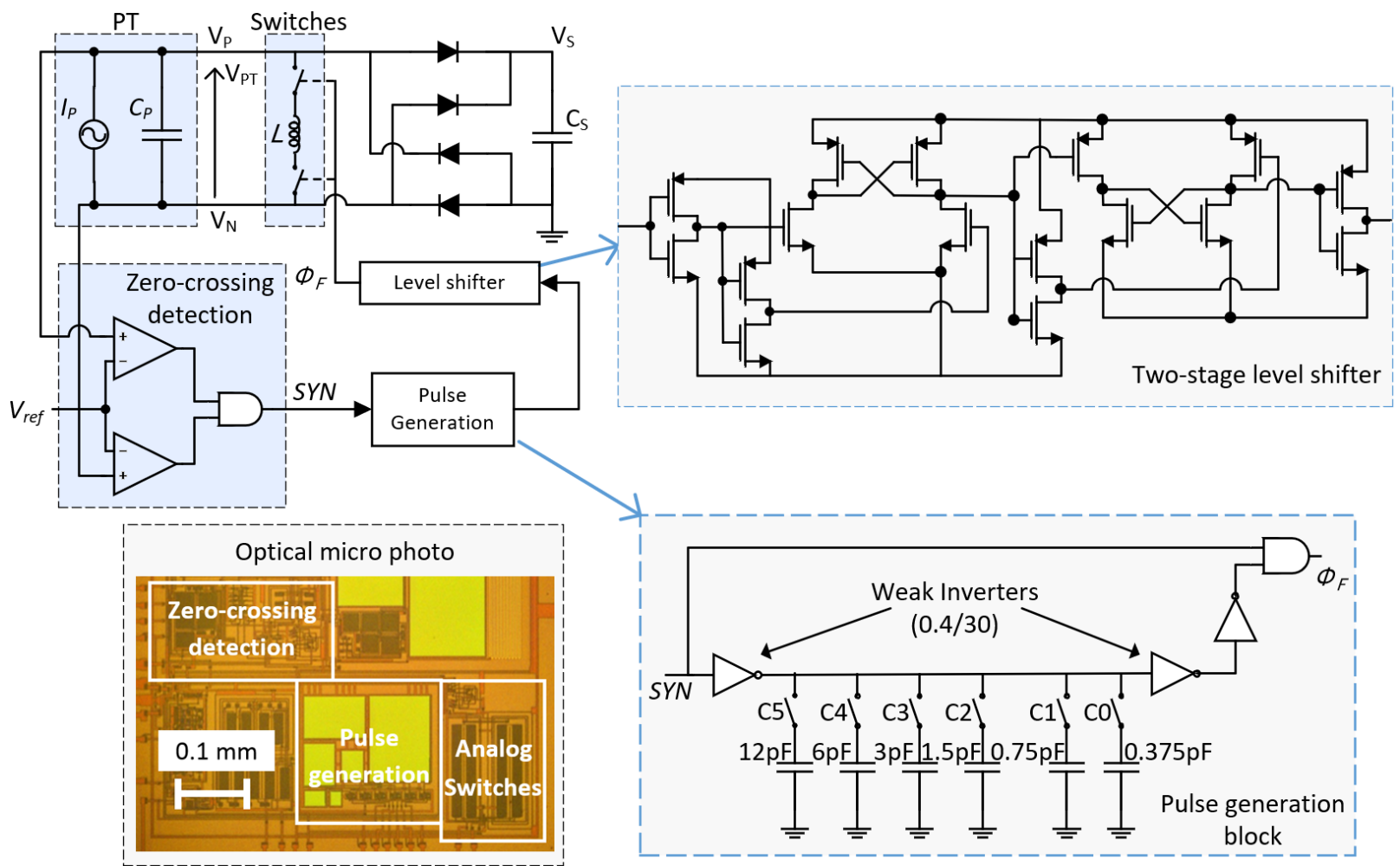

Fig. 3: SSHI circuit system architecture and circuit implementations.

to adjust the capacitance. With the help of this block, a pulse $\phi_{F}$ is generated with its pulsewidth adjusted to a half pseudoperiod of the RLC oscillation loop, which approximately equals to $2 \pi \sqrt{L C}$. This pulse signal then closes the RLC loop to flip the voltage $V_{P T}$. The next section presents the design and the modeling of a piezoelectric energy harvester in MEMS process, which is integrated with the CMOS SSHI circuit in following experiments for power measurements. The SSHI is designed and fabricated in $0.35 \mu \mathrm{m}$ HV CMOS process and the optical die photo is shown in Fig. 3. The active area of the SSHI circuit is around $0.15 \mathrm{~mm}^{2}$.

\section{MEMS VIBRATION ENERGY HARVESTER}

The MEMS vibration energy harvester employed for the integration was an in-house device developed using the most fundamental cantilever topology and an AlN (aluminum nitride) on SOI (silicon on insulator) process.

The FEA (finite element analysis) simulation of the $3.5 \mathrm{~mm}$ wide and long cantilever design is shown in figures $4 \mathrm{a}$ to $4 \mathrm{c}$. The fundamental mode resonant frequencies of the microcantilever where $50 \%$ of the beam length is occupied up by end mass is predicted at $202 \mathrm{~Hz}$ as shown in figure 4a.

The micro-cantilevers can be driven to experience approximately $300 \mathrm{MPa}$ of peak stress (a typical design limit for silicon resonators) at $120 \mathrm{~g}$ of acceleration loading on the shuttle mass and a peak displacement amplitude of about $1 \mathrm{~mm}$ as shown in figure $4 \mathrm{~b}$. This level of shuttle travel is relatively large for typical MEMS oscillators, which requires a deep cavity chip carrier to accommodate the silicon device. The induced stress across the beam length is shown in figure $4 \mathrm{c}$
The MEMS fabrication process is shown in Fig. 4d. The fabricated devices were made up of a stack of materials consisting of: $10 \mu \mathrm{m}$ thick doped silicon as the device layer with $0.5 \mu \mathrm{m}$ thick AlN piezoelectric layer on top and a further $1 \mu \mathrm{m}$ thick Al top electrode layer on top of AlN. The proof mass was achieved using un-etched regions of the $400 \mu \mathrm{m}$ thick silicon handle wafer underneath the device silicon. The device is placed in a custom laser-cut, leadless chip carrier for testing.

\section{EXPERIMENTS}

This section presents the measured output power of the MEMS cantilevered PT with a resistive load, a full-bridge rectifier (FBR) and a SSHI circuit. First, the mechanical specifications, optimal resistive load and AC output power consumed in a matched resistive load are measured. The cantilevered MEMS PT is assembled into a custom laser-cut leadless chip carrier (LCC44); the chip carrier and the MEMS cantilever are shown in Fig. 1b. During the measurements, the chip carrier containing the PT is placed on a shaker excited at the natural frequency of the PT. In order to measure the AC output power consumed in a impedance-matched resistive load, the natural frequency should first be found. Fig. 6a shows the measured open-circuit voltage amplitude, noted as $V_{O C}$, over a range of excitation frequencies. From this figure, the natural frequency of the micro cantilever is found to be around $199 \mathrm{~Hz}$. After finding the natural frequency, the PT is then excited at its resonance and connected to a variable resistor to find the optimal resistive load matching the internal impedance of the PT. The power consumed in the variable resistor is 
Eigenfrequency $=201.68$ Surface: von Mises stress (MPa) a

$\Delta 2.27$

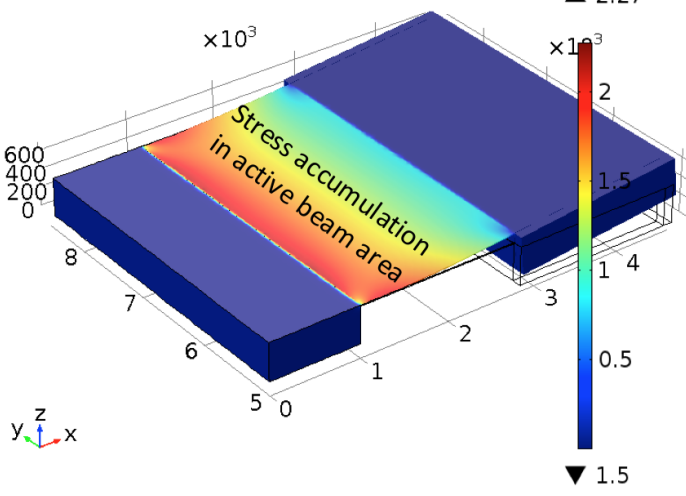

(a)

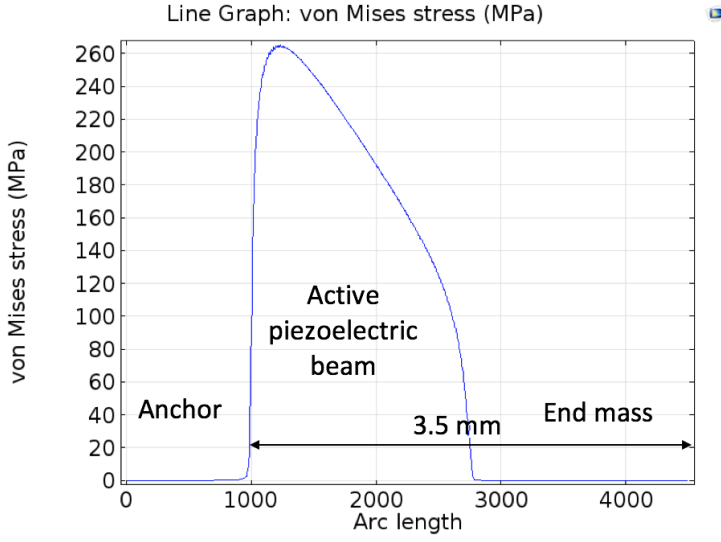

Surface: Total displacement ( $\mu \mathrm{m})$

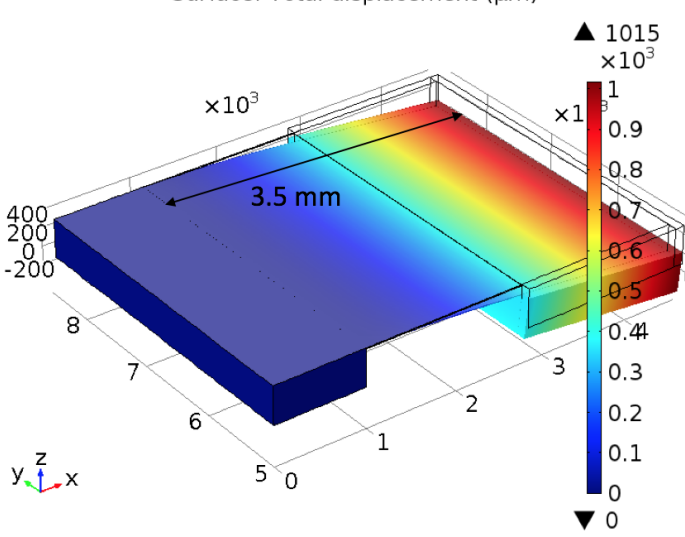

(b)

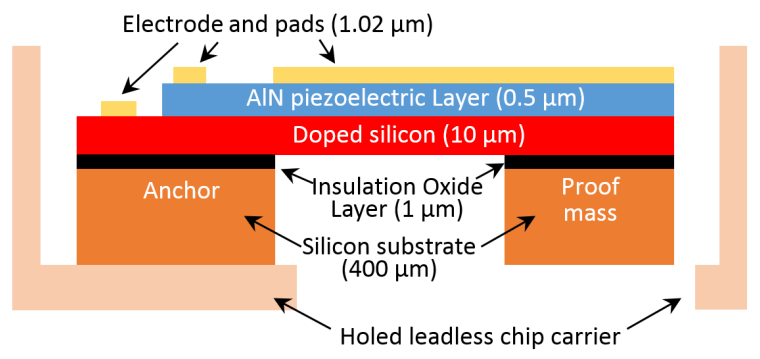

(d)

(c)

Fig. 4: (a) Fundamental resonant frequency, $202 \mathrm{~Hz}$, for the micro-cantilever (3.5 mm square) where $50 \%$ of the beam length is occupied by end mass. (b) Peak displacement of $\sim 1 \mathrm{~mm}$ for the micro-cantilevers when driven to a peak stress of $300 \mathrm{MPa}$ with $120 \mathrm{~g}$ response acceleration loading on the shuttle mass. (c) Induced stress distribution along the cantilever length. (d) A cantilevered MEMS PT with MEMS process showing different layers with corresponding thickness.

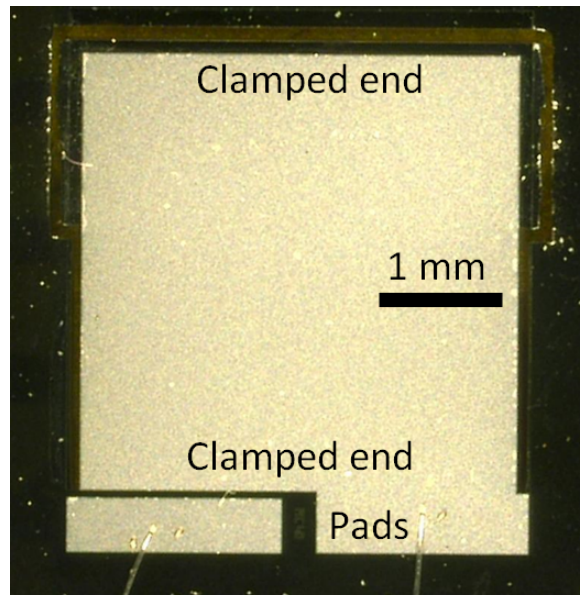

(a)

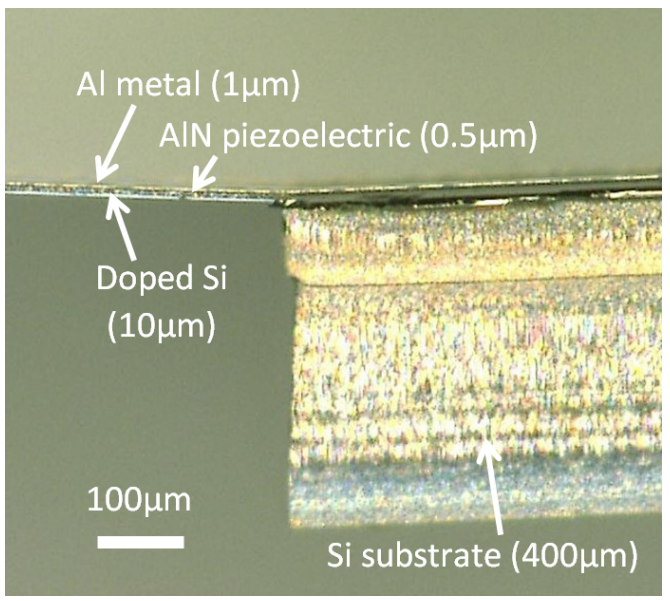

(b)

Fig. 5: (a) Optical microphoto of MEMS process with different layers. (b) Measured open-circuit voltage amplitude. 


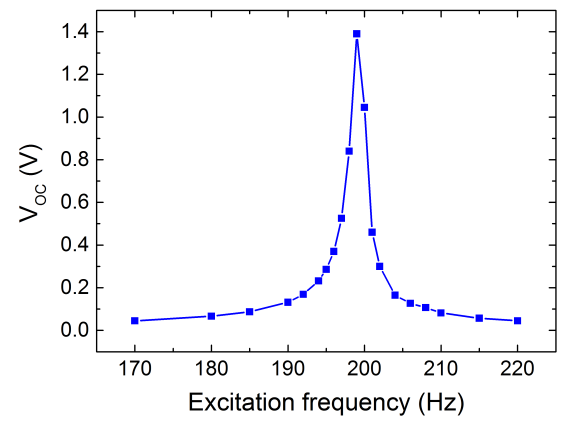

(a)

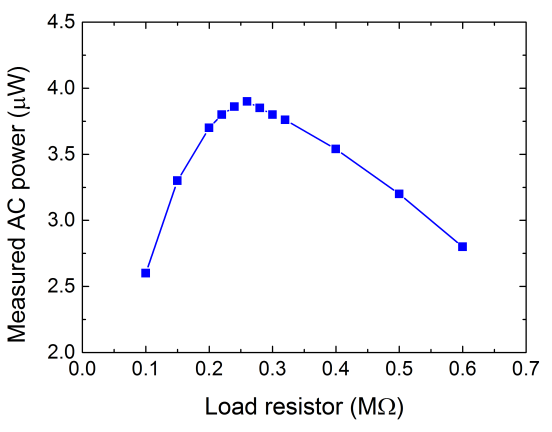

(b)

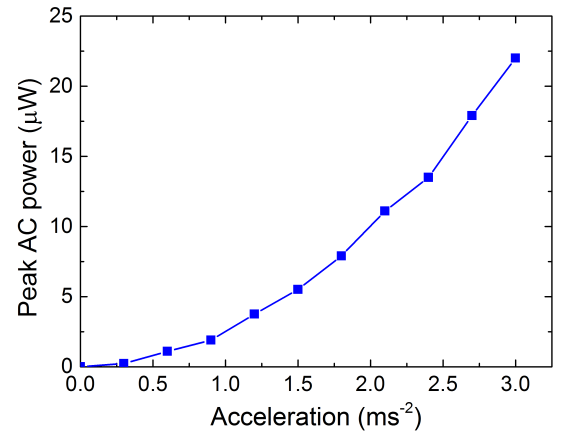

(c)

Fig. 6: (a) Measured output power in different resistive loads ( $V_{O C}=2 \mathrm{~V}$ ). (b) Measured AC peak power consumed in a $260 \mathrm{~K} \Omega$ resistive load. (c) Measured peak AC power for different acceleration levels.

calculated and the results are shown in Fig. 6b. The resistance is swept from $100 \mathrm{k} \Omega$ to $600 \mathrm{k} \Omega$ and the $\mathrm{AC}$ power is found to attain its peak while the load is at around $260 \mathrm{k} \Omega$. Then, the load resistor is kept at $260 \mathrm{k} \Omega$ by changing the excitation level to find the AC output power under different excitation levels. The results are shown in Fig. $6 \mathrm{c}$ and the peak AC output power is found to be $22.1 \mu \mathrm{W}$ at $3 \mathrm{~m} / \mathrm{s}^{2}$.

The MEMS PT is then integrated with a FBR and a SSHI circuit to measure the rectified DC power. The FBR is implemented with four off-chip Schottky diodes and the SSHI circuit is implemented in a CMOS process. The forward voltage drop of the diode is measured at around $0.2 \mathrm{~V}$. Fig. 7 shows the measured waveforms of signals $V_{P T}, S Y N$ and $\phi_{F}$ while using the FBR and using the SSHI circuit with three different inductor values. While using a passive FBR, the voltage $V_{P T}$ is slowly flipped by the generated energy to overcome the thresholds. In this case, most of generated energy is wasted and only the energy while the $V_{P T}$ attains its top and bottom limits can be extracted. In the following three figures, the SSHI circuit is employed with different inductor values. It can be seen that the voltage $V_{P T}$ is correctly flipped for this half period and the signal $S Y N$ is synchronously generated to flip $V_{P T}$. While using an inductor with higher inductance, the voltage flipping efficiency is increased, which has been studied in equation (9).

Fig. 8a shows the output power under a given excitation level while varying the output DC voltage $V_{S}$, which is the voltage across the energy storage capacitor $C_{S}$ connected at the output of the FBR and the SSHI circuit. During the measurements, the excitation level is $1.2 \mathrm{~m} / \mathrm{s}^{2}$, which corresponds to an open-circuit voltage amplitude at $V_{O C}=2 \mathrm{~V}$. Under this excitation level, the FBR can achieve its MPP (maximum power point) while $V_{S}=0.9 \mathrm{~V}$ and the peak power is $1.8 \mu \mathrm{W}$. While integrating the SSHI circuit with a $0.1 \mathrm{mH}$ inductor, the peak power is increased to $6.2 \mu \mathrm{W}$ when $V_{S}=3 \mathrm{~V}$. The peak output power achieves $8.8 \mu \mathrm{W}$, $11.6 \mu \mathrm{W}$ and $14.1 \mu \mathrm{W}$ while the inductor is chosen at $220 \mu \mathrm{H}$, $470 \mu \mathrm{H}$ and $1 \mathrm{mH}$, respectively. Compared to the FBR, the output power of integrating the SSHI with the MEMS energy harvester is increased by $3.4 \times, 4.9 \times, 6.4 \times$ and $7.8 \times$, respectively, under the $1.2 \mathrm{~m} / \mathrm{s}^{2}$ excitation with a $1 \mathrm{mH}$ inductor. According to the performance improvement equation obtained in (13), the voltage flip loss ratios for these four inductor values can be calculated as $\eta_{0.1 \mathrm{mH}} \approx 0.59, \eta_{0.22 \mathrm{mH}} \approx 0.41$, $\eta_{0.47 \mathrm{mH}} \approx 0.31$ and $\eta_{1 \mathrm{mH}} \approx 0.25$, respectively. Comparing with the waveforms obtained in Fig. 7, the measured voltage flip losses for different inductor values approximately match the theoretical values obtained with (13).

Fig. $8 \mathrm{~b}$ shows the output power under a range of excitation levels up to $3 \mathrm{~m} / \mathrm{s}^{2}$, which corresponds to an open-circuit voltage of $V_{O C}=5.2 \mathrm{~V}$. During the measurements, the output voltage is fixed at $V_{S}=4 \mathrm{~V}$. From the results, it can be seen that the FBR can only extract energy under high excitation levels from $2.5 \mathrm{~m} / \mathrm{s}^{2}$ as it sets a high threshold due to the high $V_{S}$ value. Hence, the peak output power while using a FBR is only $7 \mu \mathrm{W}$. While the SSHI circuit is employed, the output power is significantly increased to $32 \mu \mathrm{W}$ with a $100 \mu \mathrm{H}$ inductor. This value is further increased to $40.6 \mu \mathrm{W}$ with a $1 \mathrm{mH}$ inductor due to higher voltage flipping efficiency. Therefore, the SSHI circuit improves the energy extraction performance by $5.8 \times$ compared to the FBR.

Table I compares the work presented in this paper with prior publications. The second column shows the techniques used in each work and the following columns show the piezoelectric transducers used in experiments, operating frequency, internal capacitance of PTs, peak output power and the volume of PTs, respectively. The last column shows the power density $\left(\mathrm{mW} / \mathrm{cm}^{3}\right)$ by dividing the peak output power by the PT size. From the table, it can be found that the proposed the work presented in this paper shows the highest power density at $8.12 \mathrm{~mW} / \mathrm{cm}^{3}$, which is higher than all other prior cited works. The high power density is achieved due to the custom MEMS PT and the highly efficient SSHI interface circuit. The compact design of the energy harvesting system also allows it to be implemented in miniaturized self-powered systems.

\section{CONCLUSiON}

This paper presents the integration of a MEMS piezoelectric energy harvester and a CMOS SSHI interface circuit to provide a peak DC output power of $40.6 \mu \mathrm{W}$ with a record power density at $8.12 \mathrm{~mW} / \mathrm{cm}^{3}$. The piezoelectric transducer (PT) is fabricated in a MEMS process with AlN as the piezoelectric material and the CMOS circuit is implemented in a $0.35 \mu \mathrm{m}$ 


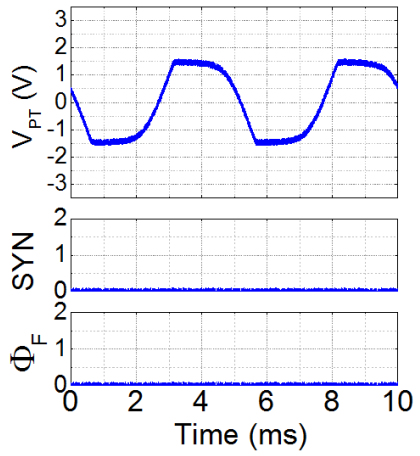

(a)

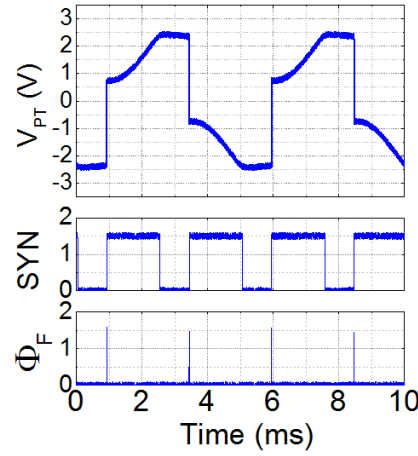

(b)

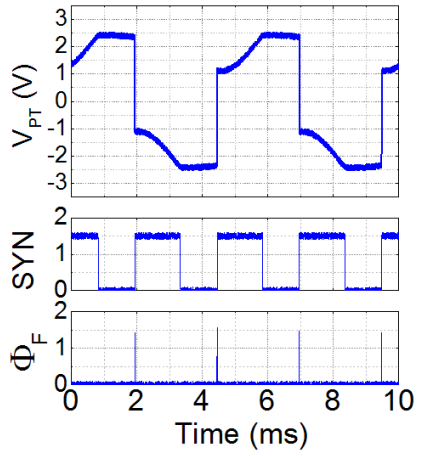

(c)

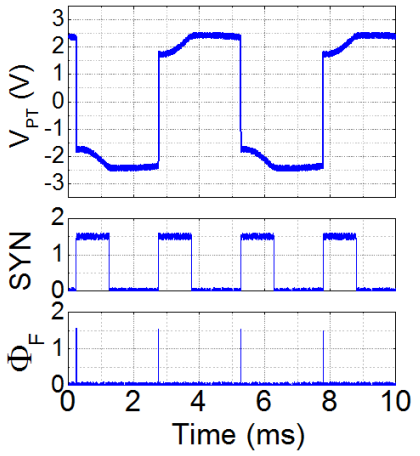

(d)

Fig. 7: (a) Full-bridge rectifier $\left(V_{O C}=2.5 \mathrm{~V}, V_{S}=1 \mathrm{~V}\right)$. (b) SSHI circuit with $100 \mu \mathrm{H}$ inductor $\left(V_{O C}=2 \mathrm{~V}, V_{S}=2 \mathrm{~V}\right)$. (c) SSHI circuit with $220 \mu \mathrm{H}$ inductor $\left(V_{O C}=2 \mathrm{~V}, V_{S}=2 \mathrm{~V}\right)$. (d) SSHI circuit with $1000 \mu \mathrm{H}$ inductor $\left(V_{O C}=2 \mathrm{~V}, V_{S}=2 \mathrm{~V}\right)$.

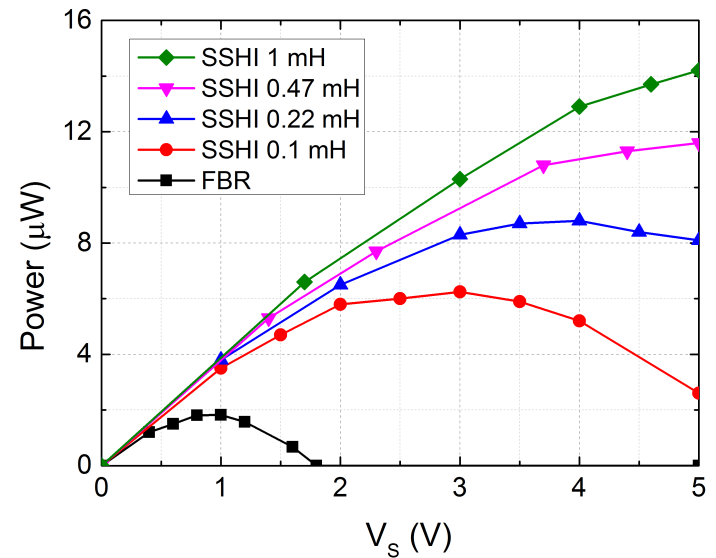

(a)

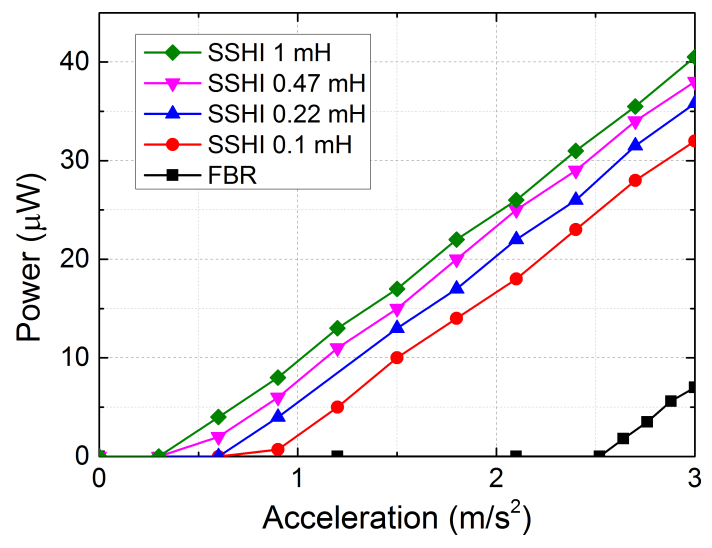

(b)

Fig. 8: (a) Measured output power using FBR and SSHI circuits with different inductor values in a range of $V_{S}$ values with $V_{O C}=2 \mathrm{~V}$. (b) Measured output power using FBR and SSHI circuits with different inductor values in a range of excitation levels from $0 \mathrm{~m} / \mathrm{s}^{2}$ to $3 \mathrm{~m} / \mathrm{s}^{2}$ with $V_{S}=4 \mathrm{~V}$.

HV (high-voltage) CMOS process. Due to the integration of a MEMS PT and a CMOS circuit, the overall system volume of an energy harvesting system can be significantly decreased to sub- $\mathrm{cm}^{3}$ scale including all off-chip components. In addition, with the MEMS PT and the CMOS circuit, the measured DC output power is as high as $40.6 \mu \mathrm{W}$ with the power improvement of $5.8 \times$ compared to a passive FBR. This demonstration shows a pathway towards miniaturized vibration-powered wireless sensor solutions as an enabling technology for the Internet of things paradigm.

\section{REFERENCES}

[1] R. J. M. Vullers, R. van Schaijk, I. Doms, C. Van Hoof, and R. Mertens, "Micropower energy harvesting," Solid-State Electronics, vol. 53, no. 7, pp. 684-693, 2009.

[2] P. D. Mitcheson, E. M. Yeatman, G. K. Rao, A. S. Holmes, and T. C. Green, "Energy harvesting from human and machine motion for wireless electronic devices," Proceedings of the IEEE, vol. 96, no. 9, pp. 14571486, 2008.

[3] S. Du, Y. Jia, C. Zhao, S.-T. Chen, and A. A. Seshia, "Real-world evaluation of a self-startup sshi rectifier for piezoelectric vibration energy harvesting," Sensors and Actuators A: Physical, vol. 264, pp. 180-187, 2017.
[4] A. Varpula, S. J. Laakso, T. Havia, J. Kyynrinen, and M. Prunnila, "Harvesting vibrational energy using material work functions," Scientific Reports, vol. 4, p. 6799, 2014.

[5] N. Rezaei-Hosseinabadi, A. Tabesh, and R. Dehghani, "A topology and design optimization method for wideband piezoelectric wind energy harvesters," IEEE Transactions on Industrial Electronics, vol. 63, no. 4, pp. 2165-2173, 2016.

[6] M. Belleville, H. Fanet, P. Fiorini, P. Nicole, M. J. M. Pelgrom, C. Piguet, R. Hahn, C. Van Hoof, R. Vullers, M. Tartagni, and E. Cantatore, "Energy autonomous sensor systems: Towards a ubiquitous sensor technology," Microelectronics Journal, vol. 41, no. 11, pp. 740-745, 2010.

[7] S. Roundy and P. K. Wright, "A piezoelectric vibration based generator for wireless electronics," Smart Materials and Structures, vol. 13, no. 5, p. 1131, 2004.

[8] S. Niu, X. Wang, F. Yi, Y. S. Zhou, and Z. L. Wang, "A universal selfcharging system driven by random biomechanical energy for sustainable operation of mobile electronics," Nat Commun, vol. 6, 2015.

[9] S. Du, Y. Jia, and A. A. Seshia, "Piezoelectric vibration energy harvesting: A connection configuration scheme to increase operational range and output power," Journal of Intelligent Material Systems and Structures, p. 1045389X16682846, 2016.

[10] S. P. Beeby, M. J. Tudor, and N. M. White, "Energy harvesting vibration sources for microsystems applications," Measurement Science and Technology, vol. 17, no. 12, p. R175, 2006.

[11] A. Harb, "Energy harvesting: State-of-the-art," Renewable Energy, vol. 36, no. 10, pp. 2641-2654, 2011.

[12] G. Tang, B. Yang, J.-q. Liu, B. Xu, H.-y. Zhu, and C.-s. Yang, "De- 
TABLE I: Performance comparison with state-of-the-art

\begin{tabular}{|c|c|c|c|c|c|c|c|}
\hline Reference & Technique & $\begin{array}{l}\text { Piezoelectric } \\
\text { transducer }\end{array}$ & Frequency & $\begin{array}{l}\text { Piezoelectric } \\
\text { capacitance }\end{array}$ & Peak power & PT size & Power density \\
\hline [37] & SSHI & Mide V22B & $225 \mathrm{~Hz}$ & $18 \mathrm{nF}$ & $68 \mu \mathrm{W}$ & $0.185 \mathrm{~cm}^{3}$ & $0.37 \mathrm{~mW} / \mathrm{cm}^{3}$ \\
\hline [34] & SSHC & Mide V21BL & $92 \mathrm{~Hz}$ & $45 \mathrm{nF}$ & $1.2 \mathrm{~mW}$ & $0.59 \mathrm{~cm}^{3}$ & $2 \mathrm{~mW} / \mathrm{cm}^{3}$ \\
\hline [46] & PSCE & Mide V22B & $173 \mathrm{~Hz}$ & $19.5 \mathrm{nF}$ & $477 \mu \mathrm{W}$ & $0.185 \mathrm{~cm}^{3}$ & $2.58 \mathrm{~mW} / \mathrm{cm}^{3}$ \\
\hline [44] & SSHI & Custom MEMS & $155 \mathrm{~Hz}$ & $8.5 \mathrm{nF}$ & $95 \mu \mathrm{W}$ & $0.027 \mathrm{~cm}^{3}$ & $3.5 \mathrm{~mW} / \mathrm{cm}^{3}$ \\
\hline$[32]$ & SSHI & Mide V22B & $134 \mathrm{~Hz}$ & $26 \mathrm{nF}$ & $500 \mu \mathrm{W}$ & $0.185 \mathrm{~cm}^{3}$ & $2.7 \mathrm{~mW} / \mathrm{cm}^{3}$ \\
\hline This work & SSHI & Custom MEMS & $199 \mathrm{~Hz}$ & $2.82 \mathrm{nF}$ & $40.6 \mu \mathrm{W}$ & $0.005 \mathrm{~cm}^{3}$ & $8.12 \mathrm{~mW} / \mathrm{cm}^{3}$ \\
\hline
\end{tabular}

velopment of high performance piezoelectric d33 mode mems vibration energy harvester based on pmn-pt single crystal thick film," Sensors and Actuators A: Physical, vol. 205, no. 0, pp. 150-155, 2014.

[13] J. Lee and B. Choi, "Development of a piezoelectric energy harvesting system for implementing wireless sensors on the tires," Energy Conversion and Management, vol. 78, no. 0, pp. 32-38, 2014.

[14] A. Khaligh, Z. Peng, and Z. Cong, "Kinetic energy harvesting using piezoelectric and electromagnetic technologies - state of the art," IEEE Transactions on Industrial Electronics, vol. 57, no. 3, pp. 850-860, 2010.

[15] S. Du, G. A. J. Amaratunga, and A. A. Seshia, "A cold-startup sshi rectifier for piezoelectric energy harvesters with increased open-circuit voltage," IEEE Transactions on Power Electronics, pp. 1-1, 2018.

[16] H. S. Kim, J.-H. Kim, and J. Kim, "A review of piezoelectric energy harvesting based on vibration," International journal of precision engineering and manufacturing, vol. 12, no. 6, pp. 1129-1141, 2011.

[17] R. Yuan and D. P. Arnold, "An input-powered vibrational energy harvesting interface circuit with zero standby power," Power Electronics, IEEE Transactions on, vol. 26, no. 12, pp. 3524-3533, 2011.

[18] J. Liang and W.-H. Liao, "Improved design and analysis of self-powered synchronized switch interface circuit for piezoelectric energy harvesting systems," IEEE Transactions on Industrial Electronics, vol. 59, no. 4, pp. 1950-1960, 2012.

[19] L. M. Miller, A. D. T. Elliott, P. D. Mitcheson, E. Halvorsen, I. Paprotny, and P. K. Wright, "Maximum performance of piezoelectric energy harvesters when coupled to interface circuits," IEEE Sensors Journal, vol. 16, no. 12, pp. 4803-4815, 2016.

[20] G. D. Szarka, B. H. Stark, and S. G. Burrow, "Review of power conditioning for kinetic energy harvesting systems," Power Electronics, IEEE Transactions on, vol. 27, no. 2, pp. 803-815, 2012.

[21] S. Du, Y. Jia, C. Zhao, G. A. J. Amaratunga, and A. A. Seshia, "A passive design scheme to increase the rectified power of piezoelectric energy harvesters," IEEE Transactions on Industrial Electronics, vol. 65, no. 9, pp. 7095-7105, 2018.

[22] Q. Shi, T. Wang, and C. Lee, "Mems based broadband piezoelectric ultrasonic energy harvester (pueh) for enabling self-powered implantable biomedical devices," vol. 6, p. 24946, 2016. [Online]. Available: http://dx.doi.org/10.1038/srep24946

[23] K. Wasa, T. Matsushima, H. Adachi, I. Kanno, and H. Kotera, "Thinfilm piezoelectric materials for a better energy harvesting mems," Microelectromechanical Systems, Journal of, vol. 21, no. 2, pp. 451457, 2012

[24] H. Liu, C. Lee, T. Kobayashi, C. J. Tay, and C. Quan, "Piezoelectric mems-based wideband energy harvesting systems using a frequency-upconversion cantilever stopper," Sensors and Actuators A: Physical, vol. 186, no. 0, pp. 242-248, 2012.

[25] L. C. J. Blystad, E. Halvorsen, and S. Husa, "Piezoelectric mems energy harvesting systems driven by harmonic and random vibrations," Ultrasonics, Ferroelectrics, and Frequency Control, IEEE Transactions on, vol. 57, no. 4, pp. 908-919, 2010.

[26] G. D. Szarka, S. G. Burrow, and B. H. Stark, "Ultralow power, fully autonomous boost rectifier for electromagnetic energy harvesters," IEEE Transactions on Power Electronics, vol. 28, no. 7, pp. 3353-3362, 2013.

[27] Y. Jia, S. Du, and A. A. Seshia, "Micromachined cantilevers-onmembrane topology for broadband vibration energy harvesting," Journal of Micromechanics and Microengineering, vol. 26, no. 12, p. 124007, 2016.
[28] X.-D. Do, H.-H. Nguyen, S.-K. Han, D. S. Ha, and S.-G. Lee, "A selfpowered high-efficiency rectifier with automatic resetting of transducer capacitance in piezoelectric energy harvesting systems," Very Large Scale Integration (VLSI) Systems, IEEE Transactions on, vol. 23, no. 3, pp. 444-453, 2015.

[29] D. Kwon and G. A. Rincon-Mora, "A single-inductor 0.35 um cmos energy-investing piezoelectric harvester," IEEE Journal of Solid-State Circuits, vol. 49, no. 10, pp. 2277-2291, 2014.

[30] G. Shi, Y. Xia, Y. Ye, L. Qian, and Q. Li, "An efficient self-powered synchronous electric charge extraction interface circuit for piezoelectric energy harvesting systems,' Journal of Intelligent Material Systems and Structures, p. 1045389X15624796, 2016.

[31] Y. Jia, S. Du, and A. A. Seshia, "Twenty-eight orders of parametric resonance in a microelectromechanical device for multi-band vibration energy harvesting," Scientific Reports, vol. 6, p. 30167, 2016.

[32] D. A. Sanchez, J. Leicht, F. Hagedorn, E. Jodka, E. Fazel, and Y. Manoli, "A parallel-sshi rectifier for piezoelectric energy harvesting of periodic and shock excitations," IEEE Journal of Solid-State Circuits, vol. 51, no. 12, pp. 2867-2879, 2016.

[33] L. Wu, X. D. Do, S. G. Lee, and D. S. Ha, "A self-powered and optimal sshi circuit integrated with an active rectifier for piezoelectric energy harvesting," IEEE Transactions on Circuits and Systems I: Regular Papers, vol. 64, no. 3, pp. 537-549, 2017.

[34] S. Du and A. A. Seshia, "An inductorless bias-flip rectifier for piezoelectric energy harvesting," IEEE Journal of Solid-State Circuits, vol. 52, no. 10, pp. 2746-2757, 2017.

[35] J. Sankman and M. Dongsheng, "A 12-uw to 1.1-mw aim piezoelectric energy harvester for time-varying vibrations with 450-na iq," Power Electronics, IEEE Transactions on, vol. 30, no. 2, pp. 632-643, 2015.

[36] H. Shen, H. Ji, J. Qiu, Y. Bian, and D. Liu, "Adaptive synchronized switch harvesting: A new piezoelectric energy harvesting scheme for wideband vibrations," Sensors and Actuators A: Physical, vol. 226, pp. 21-36, 2015.

[37] Y. K. Ramadass and A. P. Chandrakasan, "An efficient piezoelectric energy harvesting interface circuit using a bias-flip rectifier and shared inductor," IEEE Journal of Solid-State Circuits, vol. 45, no. 1, pp. 189204, 2010.

[38] S. Du, Y. Jia, and A. A. Seshia, "An efficient inductorless dynamically configured interface circuit for piezoelectric vibration energy harvesting," IEEE Transactions on Power Electronics, vol. 32, no. 5, pp. 35953609, 2017.

[39] E. Lefeuvre, A. Badel, A. Brenes, S. Seok, M. Woytasik, and C. S. Yoo, "Analysis of piezoelectric energy harvesting system with tunable sece interface," Smart Materials and Structures, vol. 26, no. 3, p. 035065, 2017. [Online]. Available: http://stacks.iop.org/0964$1726 / 26 / \mathrm{i}=3 / \mathrm{a}=035065$

[40] K. Dongwon and G. A. Rincon-Mora, "A single-inductor ac-dc piezoelectric energy-harvester/battery-charger ic converting $(0.35$ to $1.2 \mathrm{v})$ to (2.7 to 4.5v)," in Solid-State Circuits Conference Digest of Technical Papers (ISSCC), 2010 IEEE International, Conference Proceedings, pp. 494-495.

[41] M. Dini, A. Romani, M. Filippi, and M. Tartagni, "A nanopower synchronous charge extractor ic for low-voltage piezoelectric energy harvesting with residual charge inversion," IEEE Transactions on Power Electronics, vol. 31, no. 2, pp. 1263-1274, 2016.

[42] P. Gasnier, J. Willemin, S. Boisseau, G. Despesse, C. Condemine, G. Gouvernet, and J. J. Chaillout, "An autonomous piezoelectric energy 
harvesting ic based on a synchronous multi-shot technique," IEEE Journal of Solid-State Circuits, vol. 49, no. 7, pp. 1561-1570, 2014.

[43] S. Du, Y. Jia, C. D. Do, and A. A. Seshia, "An efficient sshi interface with increased input range for piezoelectric energy harvesting under variable conditions," IEEE Journal of Solid-State Circuits, vol. 51, no. 11, pp. $2729-2742,2016$.

[44] E. E. Aktakka and K. Najafi, "A micro inertial energy harvesting platform with self-supplied power management circuit for autonomous wireless sensor nodes," IEEE Journal of Solid-State Circuits, vol. 49, no. 9, pp. 2017-2029, 2014.

[45] S. Du and A. A. Seshia, "A fully integrated split-electrode synchronizedswitch-harvesting-on-capacitors (se-sshc) rectifier for piezoelectric energy harvesting with between 358enhancement," in 2018 IEEE International Solid - State Circuits Conference - (ISSCC), Conference Proceedings, pp. 152-154.

[46] T. Hehn, F. Hagedorn, D. Maurath, D. Marinkovic, I. Kuehne, A. Frey, and Y. Manoli, "A fully autonomous integrated interface circuit for piezoelectric harvesters," IEEE Journal of Solid-State Circuits, vol. 47, no. 9, pp. 2185-2198, 2012. 